\title{
Anesthesia and Cervical Dislocation Euthanasia
}

National Cancer Institute

\section{Source}

National Cancer Institute. Anesthesia and Cervical Dislocation Euthanasia. NCI

Thesaurus. Code C90355.

A method of euthanization whereby a subject is anesthetized and the spinal column is dislocated from the skull and brain. 